\title{
A Comparison of Child Abuse Potential Inventory and Parenting Stress Index with Families in the Parent-Child Interaction Therapy and Treatment as Usual Groups
}

Keri B. Whitacre

keri.whitacre@mail.wvu.edu

Follow this and additional works at: https://researchrepository.wvu.edu/etd

Part of the Psychology Commons

\footnotetext{
Recommended Citation

Whitacre, Keri B., "A Comparison of Child Abuse Potential Inventory and Parenting Stress Index with Families in the Parent-Child Interaction Therapy and Treatment as Usual Groups" (2018). Graduate Theses, Dissertations, and Problem Reports. 3726.

https://researchrepository.wvu.edu/etd/3726

This Dissertation is protected by copyright and/or related rights. It has been brought to you by the The Research Repository @ WVU with permission from the rights-holder(s). You are free to use this Dissertation in any way that is permitted by the copyright and related rights legislation that applies to your use. For other uses you must obtain permission from the rights-holder(s) directly, unless additional rights are indicated by a Creative Commons license in the record and/ or on the work itself. This Dissertation has been accepted for inclusion in WVU Graduate Theses, Dissertations, and Problem Reports collection by an authorized administrator of The Research Repository @ WVU. For more information, please contact researchrepository@mail.wvu.edu.
} 
A Comparison of Child Abuse Potential Inventory and Parenting Stress Index with Families in the Parent-Child Interaction Therapy and Treatment as Usual Groups

Keri Bailey Whitacre

Dissertation submitted to the College of Education and Human Services at West Virginia University in partial fulfillment of the requirements for the degree of Doctorate of Education

Reagan Curtis, Ph.D., Chair Cheryl McNeil, Ph.D. M Cecil Smith, Ph.D. Jessica Troilo, Ph.D.

Department of Learning Sciences and Human Development

Morgantown, West Virginia 2018

Keywords: Parent-Child Interaction Therapy, Parenting, Child Abuse and Neglect, Parenting Stress Index, Child Abuse Potential Inventory, Parental Stress Copyright 2018 Keri B. Whitacre 


\begin{abstract}
A Comparison of Child Abuse Potential Inventory and Parenting Stress Index with Families in the Parent-Child Interaction Therapy and Treatment as Usual Groups
\end{abstract}

Keri Bailey Whitacre

This study compared Parent-Child Interaction Therapy (PCIT) in a group format to a Treatment as Usual (TAU) group format within a community setting. PCIT is typically conducted individually with caregiver and child in a clinical setting. Results showed decreases on the Parenting Stress Index (PSI-SF), for both treatment groups over time with the PCIT group reporting less parental stress than the TAU group. However, there was no difference between groups over time on the Child Abuse Potential Inventory (CAPI). This study provides preliminary evidence for the efficacy of delivering PCIT in a community setting. The implication for child welfare clinicians is that an evidence-based program such as PCIT, can serve more caregivers and children and decrease parents' stress. 


\section{Acknowledgements}

I owe thanks to many people for their assistance, guidance, and encouragement throughout my graduate school. First, I would like to thank Dr. Reagan Curtis for taking me on as a graduate student and seeing this through with me. Special thanks to the other members of my committee for their time and expertise; Dr. Cheryl McNeil, Dr. M Cecil Smith, and Dr. Jessica Troilo. Dr. McNeil supervised this research project and provided mentoring that was invaluable. To Kimberly Foley--doing this research project with you was a terrific experience. I would also like to thank The Family Junction, Inc. for allowing me to serve these families. And to the undergraduate students that logged numerous hours on this project, thank you and it was a pleasure working with you.

Next, I want to say thanks to my family and friends for all the support. To my parents, John and Pam Bailey, thank you for support and encouragement that was unending; for the belief that I could do whatever I put my mind to. Mom-I wish you were still with us to see my graduation. To my mother-in-law and father-in-law for always asking how things were going. To my siblings who cared deeply about what was going on in my school process. To my dear friend Jimmy Michael, your memory motivated me many times throughout this process.

To my kids, Zack and Brady, you have endured this process and at times, had to compromise because of it. I wanted you to see that you can accomplish your goals and perseverance it crucial in life. To my husband, Seth, your support and encouragement were tremendous; thank you for all the pep talks. To honor my family, I would like to dedicate this document to them. 


\section{Introduction}

Child abuse and neglect is a serious public health concern, with an estimated 700,000 children experiencing child maltreatment annually (U.S. Department of Health \& Human Services, 2018). Children who experience abuse and neglect are more likely to have poorer mental and physical outcomes, school performance, and difficulties forming relationships (Norman et al., 2012). There are a variety of factors that influence child abuse and neglect. Maguire-Jack and Font (2017) identified a few of these risk factors as poverty, financial issues, parental stress, and parenting skills. Parenting skills have been implicated as a risk factor as caregivers may be overwhelmed when a child's behavior is inappropriate or unresponsive to their parenting tactics, thus leading to an increased probability for child abuse and neglect (Stith et al., 2009).

Risk factors for child abuse and neglect are often interrelated. For example, a parent with a high stress level or a mental health issue may not demonstrate appropriate parenting behaviors. Similarly, the Family Stress Model suggests that child behavior problems occur through severe family pressures, parental mental health, and parental efficacy (Conger, Ge, Elder, Lorenz, \& Simons, 1994). Based upon this model, stressors may impact how a family will react to stress, how they will cope with it, and what support system(s) may help them (McCubbin, Cauble, \& Patterson, 1982).

The purpose of this study was to understand the relative efficacy of a clinical laboratorybased program to reduce the potential for parental/caregiver child abuse and neglect when that program was implemented in a real-world community setting without standard laboratory equipment. This study utilized two featured programs designed to support parents and caregivers 
by reducing stress and adding positive parent-to-child behaviors expected to reduce potential for child abuse and neglect.

One way to reduce parental stress and the potential for child abuse and neglect is through parenting education/therapy. This study focused on two specific parenting programs to educate caregivers to develop parenting skills and thereby reduce family stress and the likelihood of child abuse and neglect. These two parenting programs were Parent-Child Interaction Therapy (PCIT) and Treatment as Usual (TAU). PCIT has demonstrated efficacy when implemented with individual caregiver-child dyads (Fowles, Masse, McGoron, Beveridge, Williamson, Smith, et al., 2018). The current study was part of a larger investigation conducted by Foley, McNeil, Norman, and Wallace (2016). A primary goal of the work reported here was to understand how PCIT implemented in a community group setting outside of the laboratory/clinic and without standard laboratory equipment (e.g., one-way mirrors, bug-in-ear communication) would compare to TAU regarding efficacy to reduce parenting stress and potential for child abuse and neglect.

\section{Parent-Child Interaction Therapy (PCIT)}

Eyberg developed PCIT in the 1970's based on Hanf's two-stage operant parent training model. Eyberg (1998) designed PCIT to establish a warm, loving parent-child relationship in which the parents can teach appropriate social behaviors to their children and decrease inappropriate social behaviors. PCIT is a manualized, evidence-based program to treat externalizing behavior problems in children 2 through 7 years of age (Herschell \& McNeil, 2005). PCIT consists of two distinct stages: Child-Directed Interaction (CDI) and ParentDirected Interaction (PDI). The CDI stage focuses on creating or strengthening a positive 
caregiver-child relationship, while the PDI stage focuses on decreasing inappropriate behaviors and reinforcing the child's positive behaviors (Querido, Bearss, \& Eyberg, 2004). Prior to beginning PCIT, three tasks completed by the caregiver-child dyad gather baseline relationship data to assess parenting skills (McNeil \& Hembree-Kigin, 2010). For these tasks, the caregiver should utilize their typical parenting approach to a) follow the child's lead in play, b) lead the child in play, and c) instruct the child to clean up without assistance.

Child Directed Interaction strengthens the caregiver-child relationship by drawing on positive parenting skills that contribute to increasing the child's sense of self-esteem (McNeil \& Hembree-Kigin, 2010; Bagner \& Eyberg, 2007). PCIT therapists instruct and coach caregivers on the use and delivery of their parenting instruction. The goal is to combine play therapy with differential reinforcement of other behaviors to modify their child's negative behaviors (Herschell \& McNeil, 2005). Caregivers are taught to use nondirective PRIDE skills: Praising

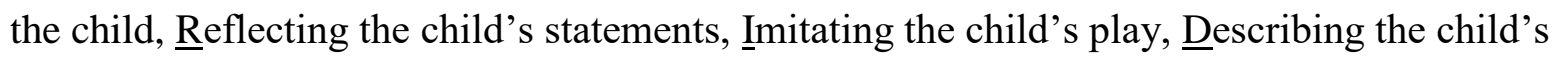
behavior, and using Enjoyment during play. By directing these PRIDE skills to the child's appropriate play, the caregiver reinforces appropriate and desired behaviors. Also during CDI, caregivers learn to eliminate behaviors referred to as QCC skills (questions, commands, and criticism). These parenting behaviors take away the child's control of their play because the caregiver is questioning, demanding, or criticizing the child rather than following the child's lead (Eyberg \& Pincus, 1999). Caregivers are coached throughout the CDI stage to reinforce appropriate behaviors while strategically ignoring inappropriate or undesired behaviors with the expectation that the frequency of negative behaviors will therefore decrease. The caregiver must demonstrate mastery of the PRIDE skills before moving to the second phase of PCIT. 
During the second stage, PDI, the caregiver leads the child's play. Caregivers often initially state three or four commands together and their child may not understand or may forget one of more of these demands. Caregivers should give clear, developmentally appropriate, effective commands and state specific consequences for noncompliance. When a caregiver gives effective commands, their child can better understand caregiver expectations and respond appropriately. CDI prepares caregivers to use description, reflection, and praise in ways that positively reinforce appropriate behaviors.

For noncompliance, the primary disciplinary technique in PDI is time-out (Herschell \& McNeil, 2005). Caregivers often struggle to implement time-out effectively. During PCIT, the therapist coaches the caregiver through the entire time-out, which lasts 3 minutes (Herschell, Calzada, Eyberg, \& McNeil, 2002a). The caregiver places the child in a pre-determined location (typically a chair in the corner) and requires the child to stay there until a timer goes off. The caregiver repeats the time-out procedure until the child complies with the original request (Querido, Bearss, \& Eyberg, 2004). Time-out is not used for all noncompliant behaviors, so other disciplinary techniques taught during PDI include use of redirection, reasoning, consequences, modeling, and praise/rewards (Eyberg \& Pincus, 1999). During PCIT sessions, therapists coach and model the delivery of positive parenting techniques for caregivers who, in turn, model appropriate behaviors for their children.

\section{PCIT Evidence-Based Characteristics}

One notable feature of PCIT is involvement of the caregiver in the therapy. The caregiver is directly involved in every step of the treatment, thus developing parenting skills, which contributes to treatment, compliance, and sustained behavioral change (Herschell, Calzada, 
Eyberg, \& McNeil, 2002b). Another feature is live coaching. Immediate feedback enables caregivers to modify their parenting skills "in the moment." Therapists observe caregiver-child interactions via a one-way mirror and provide support to caregivers if they are struggling to produce a specific PCIT skill and/or praise the child for appropriate behavior. PCIT feedback delivered via a "bug-in-the-ear" hearing device. The use of live coaching helps caregivers to become more confident in their parenting skills and achieve mastery. Another feature of live coaching is that the therapist models desired PCIT skills, providing the caregiver with example behavior to reproduce. Caregivers can ask questions, seek clarification or get additional advice. Also, the therapist and caregiver practice the PCIT skills through role-playing (Querido, Bearss, \& Eyberg, 2004). By role-playing, caregivers can see how parenting situations may unfold and brainstorm with the therapist how to respond appropriately to problem behaviors.

PCIT was designed to enable generalization of treatment behaviors from the clinical setting to other environments, such as the family home (McNeil, Eyberg, Eisenstadt, Newcomb, \& Funderburk, 1991). PCIT uses homework sessions for the caregiver and child to practice PCIT skills. Thus, desired child behaviors can be reinforced and disruptive behaviors ignored. When caregivers practice and implement PCIT skills at home, they communicate consistent expectations and enact consistent discipline. Desired, appropriate behaviors can thereby generalize to multiple environments over time (Eyberg \& Robinson, 1982).

PCIT utilizes mastery criteria for each treatment phase (Eyberg \& Funderburk, 2011). Mastery criteria for the CDI phase requires demonstrating 10 labeled praises, 10 reflections, and 10 behavioral descriptions while having fewer than three questions, commands, or criticisms during one 5-minute assessment period (Eyberg \& Pincus, 1999). Criteria for the PDI phase requires caregivers to demonstrate a minimum of 4 commands, of which $75 \%$ must be effective 
(i.e., direct, positively stated, single commands that provide an opportunity for the child to comply) and with at least $75 \%$ correct follow-through after the effective command (i.e., warning after noncompliance, labeled praise after compliance) (Eyberg \& Pincus, 1999; Herschell, Calzada, Eyberg, \& McNeil, 2002b). The caregiver must demonstrate mastery in the CDI treatment phase before proceeding to the PDI treatment phase. Then, caregivers must demonstrate PDI mastery to successfully complete PCIT (Eyberg \& Pincus, 1999; Herschell \& McNeil, 2005).

Positive outcomes for PCIT have been demonstrated in the laboratory, clinic, and inhome settings (Fowles, Masse, McGoron, Beveridge, Williamson, Smith, et al., 2018; Schuhmann, Foote, Eyberg, Boggs, \& Algina, 1998). McNeil, Eyberg, Eisenstadt, Newcomb, and Funderburk (1991) observed significant improvements for children who presented conduct problems/oppositional behavior (disobeying teacher commands, sassing, teasing, hitting, talking, whining, yelling, and breaking school rules). PCIT efficacy has been demonstrated with diverse populations including abused and neglected children (Chaffin et al., 2004), and children with developmental disorders (Bagner \& Eyberg, 2007), conduct disorders (Querido, Eyberg, \& Boggs, 2001), separation anxiety disorders (Pincus, Eyberg, \& Choate, 2005), as well as with siblings (Brestan, Eyberg, Boggs, \& Algina, 1997), adoptive children (Allen, Timmer, \& Urquiza, 2014), and with fathers as caregivers (Bagner \&Eyberg, 2003).

PCIT as Group Therapy. As described above, PCIT is implemented individually to children and caregivers in a clinical setting. However, there are advantages for conducting PCIT in a group format (Niec, Hemme, Yopp, \& Brestan, 2005). One advantage is the cost savings to families. Another advantage is that group PCIT parents are exposed to other children's and 
caregiver's behaviors and can observe how others respond to PCIT. Group participants can serve as support systems to one another.

Modification is necessary to deliver PCIT in a group format. According to Niec et al. (2005), the number of participants should be limited because of the time needed for individualized in-session coaching. PCIT session length should be increased from one to 1.5hours or 2-hours. Staffing limitations must be examined to ensure that high-quality treatment is provided. Depending upon the number of participants and children in the group, auxiliary staff may be needed to help with families while therapy is being conducted.

Community-based Parenting. Community-based parenting classes typically consist of didactic learning. Didactic learning is teacher-centered with students who sit and listen to the information being presented. Typically, participants just memorize the information for learning. According to Esler and Scriotino (1991), "Didactic lessons involve transmitting information through lecture, giving explanations and directions, answering questions, and drilling students on facts". These programs meet for a specified number of weeks and participants sit and listen to the presenter and ask questions when needed. The learning assumption is by listening and having exposure to the information, a caregiver will now implement and/or change based on the newly presented information. Historically, didactic learning has been the method of delivery for group settings because large amounts of educational material can be transferred efficiently to the students (Bligh, 2000).

\section{Purpose}

The current study was part of a larger investigation conducted by Foley, McNeil, Norman, and Wallace (2016). The purpose of the study reported here was to understand how 
PCIT implemented in a community group setting outside of the laboratory/clinic and without standard laboratory equipment (e.g., one-way mirrors, bug-in-ear communication) would compare to TAU regarding efficacy to reduce parenting stress and potential for child abuse and neglect. The study used a two-group design, comparing parenting stress and child abuse potential pre- and post-treatment. We hypothesized that both parenting stress and child abuse potential would decrease following treatment and that reduction would be larger for PCIT as compared to TAU.

\section{Method}

\section{Participants}

Forty-nine parent-child dyads utilizing a small non-profit community agency participated in this study (see Tables 1 and 2). Criteria for participation included: (1) the parent(s) was referred (or self-referred) for parenting services related to documented child physical abuse and/or neglect or perceived risk for child physical abuse or neglect; (2) the target child was 2 to 12 years of age and may or may not have been residing in the home at the time of referral; and, (3) the target child was available to be observed with the caregiver during pre- and posttreatment. Exclusion criteria included: (1) parent under the age of 18 years; (2) parent did not speak fluent English; (3) parent had suicidal ideations; and, (4) child referred for child sexual abuse.

Treatment as Usual Participants. Twenty-eight caregiver-child dyads began the TAU and twenty-four completed the treatment. The mean age of caregivers ( 23 females, 5 male) was 31.77 years $(\mathrm{SD}=7.66)$. The majority of caregivers in this group were Caucasian $(\mathrm{N}=26)$ with only one African American and one participant who did not report their race/ethnicity. Three participants were not high school graduates. Ten participants held a high school diploma or 
GED, seven had some college, one was a college graduate and one held an advanced degree.

Eight of the participants were cohabitating with domestic partners, six were married, five were separated, four were single, three were divorced, and two were re-married.

PCIT Participants. Twenty-one participants began PCIT and 20 completed treatment. The mean age of caregivers ( 18 female, 3 male) was 28.32 years $(\mathrm{SD}=6.85)$. Most caregivers in this group were Caucasian $(N=20)$, with one African American participant. Seven participants lacked a high school diploma, seven held a high school diploma or GED, six had some college, and one was a college graduate. Six participants were cohabitating with domestic partners, eight were married, two were separated, two were single, and three were divorced. 
Table 1. Adult Participant Demographic Information.

\begin{tabular}{|c|c|c|c|}
\hline & $\begin{array}{l}\text { Overall } \\
\mathrm{M}(\mathrm{SD})\end{array}$ & $\begin{array}{l}\text { PCIT } \\
\mathrm{M}(\mathrm{SD})\end{array}$ & $\begin{array}{l}\text { TAU } \\
\mathrm{M}(\mathrm{SD})\end{array}$ \\
\hline \multicolumn{4}{|l|}{ Number of Participants $(\mathrm{N})$} \\
\hline Pre-treatment & 49 & 21 & 28 \\
\hline Post-treatment & 43 & 19 & 24 \\
\hline Number of Sessions Attended & $6.96(3.19)$ & $8.10(3.18)$ & $6.11(3.00)$ \\
\hline \multicolumn{4}{|l|}{ Adult Participants } \\
\hline \multicolumn{4}{|l|}{ Gender } \\
\hline Female & 41 & 18 & 23 \\
\hline Male & 8 & 3 & 5 \\
\hline \multicolumn{4}{|l|}{ Ethnicity } \\
\hline Caucasian & 46 & 20 & 26 \\
\hline African-American & 2 & 1 & 1 \\
\hline No Reported & 1 & 0 & 1 \\
\hline Age & $30.29(7.45)$ & $28.32(6.85)$ & $31.77(7.66)$ \\
\hline Female & $29.30(6.36)$ & $27.30(6.09)$ & $30.87(6.25)$ \\
\hline Male & $35.36(10.68)$ & $34.44(9.39)$ & $35.92(12.43)$ \\
\hline \multicolumn{4}{|l|}{ Relationship } \\
\hline Cohabitating & 14 & 6 & 8 \\
\hline Divorce & 6 & 3 & 3 \\
\hline Married & 14 & 8 & 6 \\
\hline Re-Married & 2 & 0 & 2 \\
\hline Separated & 7 & 2 & 5 \\
\hline Single & 6 & 2 & 4 \\
\hline SES & $1.70(0.95)$ & $1.50(0.51)$ & $1.85(1.17)$ \\
\hline Category 1 (no occupation) & 25 & 10 & 14 \\
\hline Category 2 (unskilled labor) & 18 & 10 & 8 \\
\hline Category 3 (semi-skilled) & 1 & 0 & 1 \\
\hline Category 4 (skilled laborers) & 3 & 0 & 3 \\
\hline Category 5 (sales/clerical) & 1 & 0 & 1 \\
\hline Not Reported & 2 & 1 & 1 \\
\hline \multicolumn{4}{|l|}{ Education } \\
\hline Less than High School & 16 & 7 & 9 \\
\hline High School or GED & 17 & 7 & 10 \\
\hline Some College & 13 & 6 & 7 \\
\hline College Graduate & 0 & 1 & 1 \\
\hline Professional Degree & 1 & 0 & 1 \\
\hline
\end{tabular}


Table 2. Child Participant Demographic Information.

\begin{tabular}{clll}
\hline & Overall & PCIT & TAU \\
& $\mathrm{N}$ & $\mathrm{N}$ & $\mathrm{N}$ \\
& & & \\
\hline $\begin{array}{c}\text { Child Participants } \\
\text { Gender }\end{array}$ & 49 & 21 & 28 \\
Female & & & 13 \\
Male & 19 & 6 & 15 \\
Ethnicity & 30 & 15 & \\
Caucasian & & & 23 \\
Multiple Ethnicities & 9 & 17 & 5 \\
& 40 & 4 & $7.23(3.41)$ \\
Age & & & $7.29(3.87)$ \\
Female & $6.78(3.35)$ & $6.20(3.27)$ & \\
Male & $6.98(3.65)$ & $6.29(3.38)$ & \\
& $6.65(3.20)$ & $6.17(3.33)$ & $7.17(3.09)$ \\
& & & \\
\hline
\end{tabular}

\section{Measures}

Demographic Survey. Demographic information obtained from participants included child age, gender, and ethnicity; sibling ages and genders; maternal and paternal ages, education levels, ethnicity, relationship status, employment; and family income. Additional descriptive information collected included child and caregiver session attendance and participation, as well as homework completion. Socioeconomic status was assessed using a Two-Factor Hollingshead Index (Hollingshead, 1975).

\section{Child Abuse Potential Inventory, Fourth Edition (CAPI).}

The CAPI is a 160-item measure designed to screen and assess for physical child abuse potential. Participants with elevated abuse scores are at greater risk for committing child physical 
abuse and typically report higher stress levels. There are six narrow-band dimensions: distress, rigidity, unhappiness, problems with child and self, problems with family, and, problems with others, as well as one broad-band dimension for overall physical child abuse potential. The CAPI has a third-grade reading level with internal consistency ranging from .92 to .96 for controls and .95 to .98 for abusers (Milner, 1986). General population test-retest reliability correlations range from .91 (one-day interval) to .75 (three-month interval).

\section{Parenting Stress Index - Short Form (PSI-SF).}

The PSI-SF is a 36-item measure designed to screen and assess the level of stress in parents of children 1-month to 12 years of age (Abidin, 1995). Higher scores indicate more parenting stress. There are three narrow-band dimensions: parental distress, parent-child dysfunctional interaction, and difficult child as well as one broad-band dimension for total stress. Normal range scores would be in the $15^{\text {th }}-80^{\text {th }}$ percentile; scores above the $85^{\text {th }}$ percentile are high and have clinical significance.

\section{Procedure}

As eligible caregivers went through the intake process, they were approached about participation in a research study examining the effectiveness of PCIT. Participants who declined to participate in the study were offered treatment as usual services and no data were gathered from these individuals. The researcher explained the research project to participants, read and obtained consent prior to completing pre-treatment questionnaires, and provided participants with a copy of the consent form. The researcher assisted participants if necessary (i.e., literacy concerns) when completing questionnaires. In order to ensure confidentiality, all participants 
were assigned an identification number and all assessment measures were labeled with corresponding identification numbers. Prior to the study, participants were informed they could withdraw at any time without penalty.

Participants were assigned to PCIT or TAU groups based on availability at time of enrollment due to the need to maintain appropriate group size (i.e. attempted to keep the groups equal in number of participants). Both PCIT and TAU received weekly, two-hour group sessions over 12 weeks led by two co-therapists to ensure quality control (Chaffin et al., 2004). The 12 PCIT sessions included 6-Child Directed Interaction (CDI) and 6-Parent Directed Interaction (PDI) sessions. Participants in both treatment conditions were compensated $\$ 30.00$ for completing pre-treatment assessment measures and $\$ 70.00$ for completing post-treatment assessment measures (total of \$100.00). Pre- and post-treatment assessments took approximately 65-80 minutes.

Wierzbicki and Pekarik (1993) found attrition rates for child treatment programs to range between 40 to $60 \%$. Several methods were used to limit attrition. Participants were asked to sign an attendance contract stating that they would attend all sessions and would contact research staff if they were unable to attend. Participants received a reminder telephone call the day before the scheduled treatment session and were reminded of materials needed for the session. Participants who missed sessions could attend group or individual make-up sessions. In addition to direct financial compensation, participants received free psychotherapy services, free healthy meals and snacks during treatment sessions, free transportation to and from sessions, free child care during sessions, and toy kits for their child to keep at the completion of treatment. There was a $9.5 \%$ attrition rate for the PCIT condition $(\mathrm{N}=2)$ and a $14.5 \%$ attrition rate for the TAU condition $(\mathrm{N}$ 
$=4)$. On average, participants in the PCIT condition attended 8.10 classes $(\mathrm{SD}=3.18)$ compared to those in the TAU condition who attended 6.11 classes $(\mathrm{SD}=3.00)$.

Therapists. A licensed clinical psychologist, with expertise in PCIT, provided weekly, one-hour, group supervision throughout the study. Two graduate students and five undergraduate students served as therapists. The graduate students led PCIT and TAU groups; the undergraduate students worked with only the PCIT group. One of the graduate students had previous PCIT experience (i.e., participated on a clinical team specializing in PCIT and worked with five parent-child dyads), while the other did not. Both attended a one-week, 40-hour PCIT training workshop prior to beginning this research study and followed a detailed PCIT manual during the study. One of the graduate students had previous TAU experience (i.e., developed and modified TAU protocol and delivered TAU for the past six years) and followed a detailed TAU weekly protocol. Weekly sessions helped to maintain treatment integrity. Integrity percentages for each PCIT and TAU session were assessed using session-by-session protocol checklists (Bagner \& Eyberg, 2007; Borrego, Urqiza, Rasmussen, \& Zebell, 1999; Chaffin et al., 2004; Schuhmann et al., 1998). PCIT integrity was computed at $94 \%$ across all session and TAU integrity was $100 \%$ across all sessions.

\section{Modified Parent-Child Interaction Therapy for Community Setting.}

PCIT was provided in a community-based group setting, as opposed to the traditional individualized clinical setting. While standard PCIT protocol was followed (Eyberg \& Pincus, 1999), several adjustments were needed. First, the number of PCIT sessions was predetermined to 12 because the number of TAU sessions was 12 . Second, parents' advancement from CDI to PDI was not determined by mastery of skills. Rather all participants were advanced 
simultaneously so that treatment could be completed in 12-weeks. Third, a time-out room was not used for the target child: in standard PCIT protocol, a child who refuses to remain in the time-out chair is placed in the time-out room. The community-based facility did not permit the use of a time-out room. Therefore, if a child refused to remain in the time-out chair, the child was brought back to the time-out chair for the specified time. Fourth, direct observation of coding and coaching was conducted in the therapy room. Standard PCIT protocol requires coding and coaching to be done behind a one-way mirror with a bug-in-the ear device. Finally, a large blanket was placed on the floor to define a treatment area for the child during sessions. The child and parent remained on the blanket area during coding and coaching.

For the first 30 minutes of each two-hour session, families and PCIT staff ate dinner together. By doing so, staff could model appropriate behaviors and help participants generalize new skills to this setting. The schedule for the next 75 minutes was as follows: review homework; overview of the week's PCIT session; review PCIT mastery coding; and receive live coaching of individual PCIT skills. For homework, participants were required to practice the PCIT skills in their own home with their child. For parents who did not have custody of their child, they were to practice during supervised visits. During the overview of the weekly PCIT session, participants were introduced to the new information/skills and the therapist and participant role-played to practice the skills before coding and coaching began. Next, the caregiver completed the Eyberg Child Behavior Inventory and their demonstration of PCIT skills was coded for 5 minutes using the Dyadic Parent-Child Interaction Coding System-III. Following the coding session, each caregiver was individually coached for a minimum of 5 minutes on skills that were either leading to or preventing PCIT mastery. During the individual coding and coaching time, children played with therapeutic toys (per standard PCIT protocol). 
While this individual instruction occurred, the other PCIT group members coded and coached one another. Participants were trained how to do so and this activity enabled greater participant "buy-in" to the program. During the final 15 minutes, information from the session was reviewed and caregivers received feedback on their skill development. Homework was then assigned for the following week.

\section{Treatment as Usual Group Protocol}

Families and staff ate dinner together during the first 45 minutes of the two-hour session so that staff could model appropriate parenting behaviors and caregivers could practice these skills. During the next 60-minutes, the therapists reviewed homework, gave an overview of the session, and disseminated session information. During the final 15 minutes, provided information was reviewed and caregivers received feedback on their skill development and homework was assigned.

The treatment as usual condition consisted of 28 participants who received weekly, twohour group sessions for 12 weeks led by two therapists to ensure quality control (Chaffin et al, 2004). TAU curriculum was developed by the community agency and consisted of topics that included, but not limited to, positive discipline, natural and logical consequences, child development ages and stages, and time and stress management. One intent, and primary goal, of the TAU condition was to provide caregivers awareness of, and ideally motivation for implementation, of strategies to build healthy, positive, collaborative relationships with their children and within their families. To this end, caregivers were encouraged to share scenarios from their daily home experiences. This led to a range of topics discussed each week, most listed above, but additional topics were included based on questions/scenarios from caregivers. The 
second focus of TAU, was to provide concrete strategies for infusing positive discipline in the parent-child relationship. Both positive reinforcement and corrective discipline strategies were presented.

Overall, caregivers were given information on topics of focus and encouraged to ask questions, discuss, brainstorm, and/or constructively analyze with their specific frames of reference and situations. Caregivers welcomed the collaborative sharing of recent scenarios and offered advice regarding handling such situations in the future. Role-play was a continuous tool utilized throughout the treatment sessions. Scenarios would be explained, played out, and deconstructed based on strategies disseminated in the TAU group. The TAU model represents a didactic and somewhat interactive plan for the caregiver to build a healthy relationship with their child, address areas of weakness, and thus, reduce stress and the likelihood of negative behaviors and consequences within the parent-child relationship.

\section{Results}

\section{Preliminary and Descriptive Analyses}

Participants attended an average of 6.96 treatment sessions $(\mathrm{SD}=3.19)$. As shown in Table 3, mean pre-treatment CAPI $(\mathrm{M}=171.36, \mathrm{SD}=113.84)$ and PSI $(\mathrm{M}=88.02, \mathrm{SD}=29.37)$ scores were higher than post-treatment scores on these variables (CAPI $\mathrm{M}=154.48, \mathrm{SD}=$ 107.23; PSI $\mathrm{M}=77.02, \mathrm{SD}=21.90)$. As indicated by significant correlations in Table 3 , higher pre-CAPI scores, were related to higher post-CAPI, pre-PSI, and post-PSI scores. Post-PSI scores also were significantly related to post-CAPI scores, but other correlations were not significant. 
Table 3. Descriptive Statistics and Correlations for Key Study Variables.

\begin{tabular}{lcccc}
\hline & $M(S D)$ & Post-CAPI & Pre-PSI & Post-PSI \\
\hline Pre-CAPI & $171.36(113.84)$ & $.54^{* *}$ & $.46^{*}$ & $.44^{*}$ \\
Post-CAPI & $154.48(107.23)$ & & .10 & $.55^{* *}$ \\
Pre-PSI & $88.02(29.37)$ & & & .24 \\
Post-PSI & $77.02(21.90)$ & & & \\
\hline$* p<.01 ; * *<<.001$ & & & & \\
\hline
\end{tabular}

The 7\% missing data were missing completely at random based on a non-significant Little's MCAR test, and skewness and kurtosis were appropriate to assume normality (Field, 2009). Independent samples t-tests showed no statistically significant difference between PCIT and TAU groups at pre-test. Costello, Shook, Wallace, and McNeil (2018) found that demographic variables were not related to key study variables and key demographic variables in this study were not correlated with outcome measures, so demographics were not included as covariates. A series of mixed model ANOVAs determined the effect of time (pre vs. post) and treatment (PCIT vs. TAU) on each outcome variable (see Table 4). Levene's test for homogeneity of variance was significant for post CAPI scores $(\mathrm{p}=.014)$ so the GreenhouseGeisser correction was utilized in that mixed-model ANOVA.

\section{Child Abuse Potential.}

A mixed model ANOVA was conducted on broad band CAPI scores to determine the effect of time and treatment group on child abuse potential. There were elevated Lie scale scores post-PCIT group as compared to post-TAU, $\mathrm{t}(42)=2.53, \mathrm{p}=.015$, as well as a statistically significant correlation pre-to post-treatment in Lie scale scores $(r=.35, \mathrm{p}=.024)$ so the Lie scale 
was included as a covariate. There was a non-significant main effect of time, although CAPI changes over time approached significance, $F(1,40)=3.49, p=.07, \eta^{2}=.005$. There was a statistically significant main effect for treatment group, $F(1,39)=4.92, p=.03, \eta^{2}=.11$. When controlling for elevated Lie scales at post-treatment, there was a non-significant interaction between treatment group and time, indicating that changes in child abuse did not significantly differ between the PCIT and TAU groups, $F(1,39)=1.24, p=.273, \eta^{2}=.03$. See Table 4. Given this non-significant interaction, narrow band CAPI scores were not analyzed further.

Table 4. Mixed Model ANOVAs for Treatment Predictors.

\begin{tabular}{llll}
\hline \multicolumn{1}{c}{ Predictor } & $F$ & $p$ & partial $\eta^{2}$ \\
\hline Child Abuse Potential & & & \\
Time & 3.49 & 0.07 & 0.005 \\
Treatment Group & 4.92 & 0.03 & 0.11 \\
Time x Treatment Group & 1.24 & 0.27 & 0.03 \\
& & & \\
Total Parenting Stress & & & \\
Time & 9.57 & 0.004 & 0.189 \\
Treatment Group & 0.01 & 0.92 & .001 \\
Time x Treatment Group & 4.82 & 0.03 & 0.11 \\
& & & \\
Parental Distress & & & \\
Time & 14.5 & 0.001 & 0.26 \\
Treatment Group & 0.16 & 0.69 & .001 \\
Time x Treatment Group & 11.3 & 0.002 & 0.22 \\
& & & \\
Parent-Child Dysfunction & & & \\
Time & 4.27 & 0.045 & 0.094 \\
Treatment Group & 0.15 & 0.70 & .004 \\
Time x Treatment Group & 5.82 & 0.02 & 0.12 \\
& & & \\
Difficult Child & & & \\
Time & 7.56 & 0.009 & 0.156 \\
Treatment Group & 0.06 & 0.81 & .001 \\
Time x Treatment Group & 0.33 & 0.57 & 0.008 \\
\hline
\end{tabular}




\section{Parenting stress.}

A mixed model ANOVA was conducted to determine change over time and treatment group differences in broad band parenting stress scores on the PSI. There was a statistically significant main effect of time, $F(1,41)=9.57, p=.004, \eta^{2}=.189$, non-significant main effect for treatment group, $F(1,41)=.01, p=.92, \eta^{2}<.001$, and a statistically significant interaction between treatment group and time, $F(1,41)=4.82, p=.03, \eta^{2}=.11$. Individuals in the PCIT treatment group demonstrated larger reductions in parenting stress pre- $(\mathrm{M}=96.26, \mathrm{SD}=33.74)$ to post-treatment $(M=71.21, \mathrm{SD}=17.58)$. See Table 4 . This significant interaction in broad band parenting stress was examined further through narrow band scale scores.

Parental distress. A mixed model ANOVA was conducted to determine change over time and treatment group differences in parental distress scores on the PSI (see Table 4). There was a statistically significant main effect for time, with distress decreasing from pre- to posttreatment, $F(1,41)=14.48, p<.001, \eta^{2}=.26$, a non-significant main effect for treatment group, $F(1,41)=.16, p=.69, \eta^{2}<.001$, and a statistically significant interaction between treatment group and time, $F(1,41)=11.25, p=.002, \eta^{2}=.22$. Individuals in the PCIT treatment group demonstrated larger reductions in parental distress from pre- $(\mathrm{M}=34.16, \mathrm{SD}=9.29)$ to posttreatment $(\mathrm{M}=23.58, \mathrm{SD}=6.52)$. See Table 4 .

Parent-child dysfunction. A mixed model ANOVA was conducted to determine change over time and treatment group differences in parent-child dysfunction scores on the PSI (see Table 4). There was a statistically significant main effect of time, with parent-child dysfunction decreasing over time, $F(1,41)=4.27, p=.045, \eta^{2}=.094$. There was a non-significant main effect for treatment group, $F(1,41)=.15, p=.70, \eta^{2}=.004$. There was a statistically significant interaction between treatment group and time, $F(1,41)=5.82, p=.02, \eta^{2}=.12$, with individuals 
in the PCIT treatment group demonstrating larger reductions pre- $(\mathrm{M}=28.11, \mathrm{SD}=14.13)$ to post-treatment $(\mathrm{M}=20.05, \mathrm{SD}=5.32)$.

Difficult child. A mixed model ANOVA was conducted to determine change over time and treatment group differences in difficult child scores on the PSI (see Table 4). There was a statistically significant main effect of time, with difficult child decreasing over time, $F(1,41)=$ $7.56, p=.009, \eta^{2}=.156$. There was not a significant main effect of treatment group, $F(1,41)=$ $.06, p=.81, \eta^{2}=.001$, nor was there any significant interaction effect.

\section{Discussion}

The purpose of this study was to understand how PCIT implemented in a community group setting outside of the laboratory/clinic and without standard laboratory equipment (e.g., one-way mirrors, bug-in-ear communication) would compare to TAU regarding efficacy to reduce parenting stress and potential for child abuse and neglect. The study used a two-group design, comparing parenting stress and child abuse potential pre- and post-treatment for each group. We hypothesized that both parenting stress and child abuse potential would decrease following treatment and that reduction would be larger for PCIT as compared to TAU.

PCIT was modified so that there was no one-way mirror for observing parent-child interactions, and no "bug-in-the-ear" piece for the therapist to prompt parents' behaviors in real time. There was also no time-out room. All three have been considered essential components of laboratory/clinic-based PCIT. Other components that were modified included the length of services and the criteria for advancing to the next level when PCIT skills were mastered. Typically, PCIT is conducted in a clinical setting with a parent and child dyad desiring therapy services. The population of this study was court-ordered due to being at risk for child abuse and 
neglect, having been affected by child abuse and neglect, or having a substantiated child abuse and neglect report.

There was a statistically significant decrease in parenting stress, as shown by PSI scores, from pre- to post-treatment. The PCIT group showed a greater decrease than did the TAU group. Participants developed skills that helped them cope better with the demands of parenting. Two of the three subscales of the PSI, parental distress and parent-child dysfunction, decreased significantly more in the PCIT as compared to the TAU. PCIT specifically focuses on the parentchild relationship and this could account for the observed decrease in scores. By providing caregivers with psychosocial education regarding their children's behaviors, caregivers may better understand and be able to discuss inappropriate behaviors with their child, thereby alleviating stress. Developmentally appropriate behavior expectations were taught to the caregivers, thus perhaps changing any unrealistic behavioral expectations regarding their children's behavior, and reducing risks of child abuse and neglect. Modeling and coaching caregivers on effective behavior management strategies may have led to decreases in parental stress. As mentioned previously, the Family Stress Model (Conger, et al., 1994) suggests that child behavior problems occur through family stress. When caregivers receive treatment, the negative effects of parental stress appear to decrease. Helping the caregiver acquire new parenting and coping skills seem to contribute to the decrease in stress. By having therapy services in a group format, caregivers may also have found support from other caregivers, which might also have served to decrease their stress.

Contrary to the hypothesis, there was not a significant decrease in child abuse potential scores on the CAPI, from pre- to post-treatment. This result was not expected, as other studies have shown that participants with a history of child abuse and neglect who received PCIT 
treatment reported decreases in potential child abuse and neglect following treatment (Borrego et al., 1999; Chaffin, Funderburk, Bard, Valle, \& Gurwitch, 2011; Chaffin et al., 2004; Thomas, \& Zimmer-Gembeck, 2011; Thomas, \& Zimmer-Gembeck, 2012). Deviations from standard PCIT protocol may have contributed to the lack of change in child abuse and neglect scores. Another possibility may be more treatment time is needed for these caregivers. Based on the Family Stress Model, these families, after receiving treatment and having a support system, should show a decrease in stress.

A couple of limitations were observed during this study. TAU did not involve active participation to develop parenting skills (parents did not have to practice or model new parenting techniques during the sessions). Consequently, the researchers questioned whether this TAU used in this study adheres to the standard model TAU in a community setting.

Small sample sizes for treatment groups may have affected the results. This study was successful in retaining the majority of participants, but more participants would have made the study stronger. For future research, a larger sample size is needed.

Another limitation was that not all caregivers participated in provided therapy services. For example, if only the mother had custody of the child, then only the mother and child participated, and not the child's father. The participation of the parent and child was expected to yield positive results, but may not result in changes to the relationship with the other parent because of his or her lack of involvement. Including the entire family in treatment services would be an improvement for future research.

\section{Conclusion}


This study demonstrated positive results for the implementation of PCIT outside of the laboratory or clinical setting. Group PCIT was delivered in a community setting, with modifications, and the findings suggest that PCIT can be effective in such settings. The implication for child welfare clinicians is that an evidence-based program such as PCIT, can serve more caregivers and children and decrease parents' stress. Another finding of this study, is PCIT showed positive results with the court-ordered population. In addition to the treatment of PCIT, we found weekly phone calls, transportation to treatment sessions, free healthy dinners, free childcare, follow-up phone calls, and rapport to be integral pieces for success. The implication for human service workers is that PCIT can show positive results with the courtordered population. Another critical point to mention is a goodness of fit between the courtordered population and child welfare clinicians. This is important for building a rapport and gaining the trust of the caregiver, thus allowing for treatment services to be effective and the mutual goal of helping the child and family achieved. Further research is needed to pinpoint the specific treatment components of PCIT that lead to decreases in parental stress and child abuse and neglect potential. 


\section{References}

Abidin, R.R. (1995). Parenting stress index, $3^{\text {rd }}$ Ed. Psychological Assessment Resources, Inc. Lutz, FL.

Allen, B., Timmer, S., \& Urquiza, A. (2014). Parent-child interaction therapy as an attachment-based intervention: Theoretical rationale and pilot data with adopted children. Children and Youth Services Review, 47, 334-341.

Bagner, D.M., \& Eyberg, S.M. (2003). Father involvement in parent training: When does it matter? Journal of Clinical Child and Adolescent Psychology, 32, 599-605.

Bagner, D.M., \& Eyberg, S.M. (2007). Parent-child interaction therapy for disruptive behavior in children with mental retardation: A randomized controlled trial. Journal of Clinical Child and Adolescent Psychology, 36, 418-429.

Bligh, D.A. (2000). What's the Use of Lectures? San Franciso: Jossey-Bass.

Borrego, J.J., Urquiza, A.J., Rasmussen, R.A., \& Zebell, N. (1999). Parent-child interaction therapy with a family at high risk for physical abuse. Child Maltreatment, 4(4) 331-342.

Brestan, E.V., Eyberg. S.M., Boggs, S.R., \& Algina, J. (1997). Parent-child interaction therapy: Parents' perceptions of untreated siblings. Child \& Family Behavior Therapy, 19, 13-28.

Chaffin, M., Funderburk, B., Bard, D., Valle, L., \& Gurwitch. R. (2011). A combined motivation and parent-child interaction therapy package reduces child welfare recidivism in a randomized dismantling field trial. Journal of Consulting and Clinical Psychology, 79(1), 84-95. 
Chaffin, M., Silovsky, J.F., Funderburk, B., Valle, L.A., Brestan, E.V., Balachova, T., Jackson, S., Lensgraf, J., \& Bonner, B.L. (2004). Parent-child interaction therapy with physical abusive parents: Efficacy for reducing future abuse reports. Journal of Consulting and Clinical Psychology, 72, 500-510.

Conger, R.D., Ge, X., Elder, G.H., Lorenz, F.O., \& Simons, R.L. (1994). Economic stress, coercive family process, and developmental problems of adolescents. Child Development, $65,541-561$.

Costello, A.H., Shook, N.J., Wallace, N.M., \& McNeil, C.B. (2018). Examining factors associated with elevated Lie Scale responding on the Child Abuse Potential Inventory. Child Abuse \& Neglect, 76, 56-64.

Esler, W., \& Sciortino, P. (1991). Methods of teaching. An overview of current practices. Raleigh, NC: Contemporary Publishing Company, Inc.

Eyberg, S.M. (1988). Parent-child interaction therapy: Integration of traditional and behavioral concerns. Child \& Family Behavior Therapy, 10, 33-46.

Eyberg, S.M., \& Funderburk, B. (2011). Parent-child interaction therapy treatment protocol. Gainesville, FL: PCIT International.

Eyberg, S., \& Pincus, D. (1999). Eyberg child behavior inventory and Sutter-Eyberg student behavior inventory - Revised. Psychological Assessment Resources, Odessa, DL: Psychological Assessment Resources.

Eyberg, S.M., \& Robinson, E.A. (1982). Parent-child interaction training: Effects on family functioning. Journal of Clinical Child Psychology, 11, 2, 130-137.

Field, A. (2009). Discovering statistics using SPSS. Sage publications. Thousand Oaks, CA. Foley, K., McNeil, C.B., Norman, M., \& Wallace, N.M. (2016). Effectiveness of group format 
parent-child interaction therapy compared to treatment as usual in a community outreach organization. Child \& Family Behavior Therapy, 38, 4, 279-298.

Fowles, T.R., Masse, J.J., McGoron, L., Beveridge, R.M., Williamson, A.A., Smith, M.A., \& Parrish, B.P. (2018). Home-based versus clinic-based parent-child interaction therapy: Comparative effectiveness in the content of dissemination and implementation. Journal of Child \& Family Studies, 27, 1115-1129.

Herschell, A.D., Calzada, E.J., Eyberg, S.M., \& McNeil, C.B. (2002a). Parentchild interaction therapy: New directions in research. Cognitive and Behavioral Practice, 9, 9-16.

Herschell, A.D., Calzada, E.J., Eyberg, S.M., \& McNeil, C.B. (2002b). Clinical issues in parent-child interaction therapy. Cognitive and Behavioral Practice, 9, 16-27.

Herschell, A.D., \& McNeil, C.B. (2005). Theoretical and empirical underpinnings of parent-child interaction therapy with child physical abuse populations. Education and Treatment of Children, 28, 142-162.

Hollingshead, A. (1975). Four factor index of social status. Unpublished manuscript. New Haven, CT: Yale University

Maguire-Jack, K. \& Font, S.A. (2017). Community and Individual Risk Factors for Physical Child Abuse and Child Neglect: Variations by Poverty Status. Child Maltreatment, 22(3), 215-226.

McCubbin, H.L., Cauble, A.E., \& Patterson, J.M. (1982). Family stress, coping, and social support. Thomas Books, Springfield, IL.

McNeil, C.B., Eyberg, S., Eisenstadt, T.H., Newcomb, K., \& Funderburk, B. (1991). 
Parent-child interaction therapy with behavior problem children: Generalization of treatment effects to the school setting. Journal of Clinical Child Psychology, 20, 140-151.

McNeil, C.B., \& Hembree-Kigin, T.L. (2010). Parent-child interaction therapy $2^{\text {nd }}$ ed. Springer Publishers, New York City, NY.

Milner, J.S. (1986). CAP inventory manual, $2^{\text {nd }}$ Ed., An interpretive manual for the CAP inventory. Psychological Assessment Resources, Lutz, FL.

Niec, L.N., Hemme, J.M., Yopp, J.M., \& Brestan, E.V (2005). Parent-child interaction therapy: The rewards and challenges of a group format. Cognitive and Behavioral Practice, 12, 113-125.

Norman, R.E., Byambaa, M., De, R., Butchart, A., Scott, J., \& Vos, T. (2012). The long-term health consequences of child physical abuse, emotional abuse, and neglect: a systematic review and meta-analysis. PLoS medicine, 9(11), e1001349.

Pincus, D.B., Eyberg, S.M., \& Choate, M.L. (2005). Adapting parent-child interaction therapy for young children with separation anxiety disorder. Education and Treatment of Children, 28, 163-181.

Querido, J.G., Eyberg, S.M., \& Boggs, S.R. (2001). Revisiting the accuracy hypothesis in families of young children with conduct problems. Journal of Clinical Child Psychology, $30,253-261$.

Querido, J.G., Bearss, K., \& Eyberg, S.M. (2004). Parent/child interaction therapy. In F.W. Kaslow, \& T. Patterson (Eds.), Comprehensive handbook of psychotherapy (pp. 91-113). Wiley; Hoboken, NJ.

Schuhmann, E.M., Foote, R.C., Eyberg, S.M., Boggs, S.R., \& Algina, J. (1998). 
Efficacy of parent-child interaction therapy: Interim report of a randomized trial with short-term maintenance. Journal of Clinical Child Psychology, 27, 34-45.

Stith, S., Ting Liu, L., Davies, L.C., Boykin, E.L., Alder, M.C., Harris, J.M, et al. (2009). Risk factors in child maltreatment: A meta-analytic review of the literature. Aggression and Violent Behavior, 14, 13-29.

Thomas, R., \& Zimmer-Gembeck, M. (2011). Accumulating evidence for parent-child interaction therapy in the prevention of child maltreatment. Child Development, 82, 177192.

Thomas, R., \& Zimmer-Gembeck, M. (2012). Parent-child interaction therapy: An evidencebased treatment for child maltreatment. Child Maltreatment, 17, 253-266.

U.S. Department of Health \& Human Services, Administration for Children and Families, Administration on Children, Youth and Families, Children's Bureau. (2018). Child maltreatment 2016. Available from https://www.acf.hhs.gov/cb/research-datatechnology/statistics-research/child-maltreatment.

Wierzbicki, M., \& Pekarik. G. (1993). A meta-analysis of psychotherapy dropout. Professional Psychology: Research and Practice, 24, 190-195. 